\title{
Iterative Learning Control of Hysteresis in Piezoelectric Actuators
}

\author{
Guilin Zhang, ${ }^{1}$ Chengjin Zhang, ${ }^{2}$ and Chaoyang Wang ${ }^{3}$ \\ ${ }^{1}$ College of Electrical Engineering and Automation, Shandong University of Science and Technology, Qingdao 266590, China \\ ${ }^{2}$ School of Mechanical, Electrical and Information Engineering, Shandong University at Weihai, Weihai 264209, China \\ ${ }^{3}$ College of Mathematics and Systems Science, Shandong University of Science and Technology, Qingdao 266590, China \\ Correspondence should be addressed to Chengjin Zhang; cjzhang@sdu.edu.cn
}

Received 2 April 2014; Accepted 8 May 2014; Published 25 May 2014

Academic Editor: Qingsong Xu

Copyright (c) 2014 Guilin Zhang et al. This is an open access article distributed under the Creative Commons Attribution License, which permits unrestricted use, distribution, and reproduction in any medium, provided the original work is properly cited.

We develop convergence criteria of an iterative learning control on the whole desired trajectory to obtain the hysteresiscompensating feedforward input in hysteretic systems. In the analysis, the Prandtl-Ishlinskii model is utilized to capture the nonlinear behavior in piezoelectric actuators. Finally, we apply the control algorithm to an experimental piezoelectric actuator and conclude that the tracking error is reduced to $0.15 \%$ of the total displacement, which is approximately the noise level of the sensor measurement.

\section{Introduction}

Piezoelectric actuators (PEAs) have been widely used in nanopositioning systems due to their fast response and nanometer scale resolution [1-3]. However, the hysteresis existing in PEAs can greatly limit system performance $[4,5]$. Control of hysteretic system is an important area of control system research and a challenging problem [6-9]. Research on feedback and model-based feedforward control has been studied to achieve relatively high-precision positioning [10-13]. Iterative methods can be used to improve the positioning performance if the positioning application is repetitive. Therefore, many researchers study the iterative and adaptive control methods to minimize the adverse effect of hysteresis [14-18].

The main challenge in iterative approaches for hysteretic systems is to assure convergence of the iterative algorithm. Leang and Devasia divide a general desired trajectory into some monotonicity partition $[15,16]$. Afterwards, they prove the convergence of iterative learning control (ILC) algorithm on each single branch. In this paper, we study the design of (ILC) algorithm to compensate for hysteresis-caused error in PEAs. The main contribution of our work is proving convergence of ILC algorithm on whole tracking trajectory.

The remainder of this paper is organized as follows. First, we state the problem in the next section. Afterwards, we briefly review the Prandtl-Ishlinskii model in the context of this work and prove convergence of the ILC algorithm we designed. Finally, we implement the ILC algorithm on experimental stage and show our experimental results and conclusions.

\section{Problem Statement}

Consider a hysteretic system of the following form:

$$
y(t)=H[v(t)],
$$

where $v(t) \in \mathbb{R}$ is the input, $y(t) \in \mathbb{R}$ is the output, and $H$ denotes the hysteresis function $\mathbb{R} \rightarrow \mathbb{R}$. For a given desired trajectory $y_{d}(t)$ defined on the finite time interval $t \in[0, T]$, the objective is to find an input $v_{d}(t)$ by way of the following iterative learning control (ILC) algorithm:

$$
v_{k+1}(t)=v_{k}(t)+\gamma e_{k}(t),
$$

where $e_{k}(t)=y_{d}(t)-y_{k}(t), \gamma$ is a constant (to be determined), and $v_{k}(t)$ and $y_{k}(t)$ are the input and output at the $k$ th iteration, respectively. Figure 1 depicts the block diagram of the ILC algorithm. The goal of the ILC algorithm is to generate 


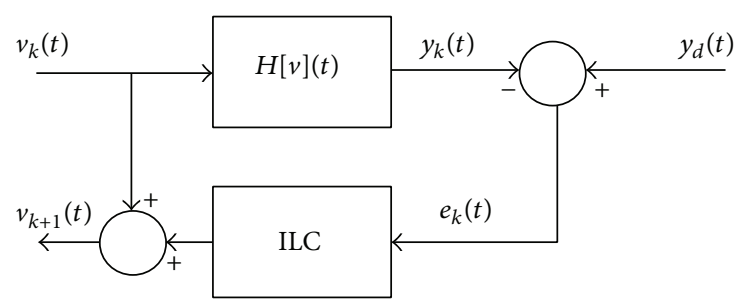

Figure 1: The ILC scheme.

a feedforward control input $v_{k}(t) \rightarrow v_{d}(t)$ in the $\|\cdot\|_{\infty}$ norm sense, where

$$
\|\cdot\|_{\infty} \triangleq \sup _{t \in[0, T]}|v(t)| .
$$

In this paper, $C[0, T]$ is used to denote the space of continuous functions on $t \in[0, T]$, and $C_{m}[0, T]$ denotes the space of continuous monotone functions on $t \in[0, T]$.

Definition 1 (incremental strictly increasing operators). An operator $F: C[0, T] \rightarrow C[0, T]$ is called incremental and strictly increasing, if, for $v_{1}, v_{2} \in C[0, T]$, considering the ordering $v_{1}(t) \geq v_{2}(t)$ for all $t \in[0, T]$, there exist constants $k_{1}, k_{2}>0$ such that

$$
\begin{aligned}
k_{1}\left(v_{1}(t)-v_{2}(t)\right) & \leq F\left[v_{1}\right](t)-F\left[v_{2}\right](t) \\
& \leq k_{2}\left(v_{1}(t)-v_{2}(t)\right),
\end{aligned}
$$

for any $t \in[0, T]$.

Lemma 2. Let the operator $F: C[0, T] \rightarrow C[0, T]$ be incremental and strictly increasing. If $0<\gamma \leq 1 / k_{2}$ and $v_{0}(t) \leq$ $v_{d}(t)$ in (2) for all $t \in[0, T]$, then $v_{k}(t) \leq v_{d}(t)$ for any $t \in$ $[0, T]$.

Proof. We use the mathematical induction to prove this assertion. First, we prove $v_{d}(t)-v_{1}(t) \geq 0$ for any $t \in[0, T]$. Consider

$$
\begin{aligned}
v_{d}(t) & -v_{1}(t) \\
& =v_{d}(t)-v_{0}(t)-\gamma\left(y_{d}(t)-y_{0}(t)\right) \\
& =v_{d}(t)-v_{0}(t)-\gamma\left(F\left[v_{d}\right](t)-F\left[v_{0}\right](t)\right) \\
& \geq v_{d}(t)-v_{0}(t)-\gamma k_{2}\left(v_{d}(t)-v_{0}(t)\right) \\
& =\left(1-\gamma k_{2}\right)\left(v_{d}(t)-v_{0}(t)\right),
\end{aligned}
$$

where $1-\gamma k_{2} \geq 0$. We can obtain $v_{d}(t) \geq v_{1}(t)$. Then, we assume $v_{d}(t)-v_{k}(t) \geq 0$, for all $t \in[0, T]$, and

$$
\begin{aligned}
v_{d}(t) & -v_{k}+1(t) \\
& =v_{d}(t)-v_{k}(t)-\gamma\left(y_{d}(t)-y_{k}(t)\right) \\
& =v_{d}(t)-v_{k}(t)-\gamma\left(F\left[v_{d}\right](t)-F\left[v_{k}\right](t)\right) \\
& \geq v_{d}(t)-v_{k}(t)-\gamma k_{2}\left(v_{d}(t)-v_{k}(t)\right) \\
& =\left(1-\gamma k_{2}\right)\left(v_{d}(t)-v_{k}(t)\right) ;
\end{aligned}
$$

we also get $v_{d}(t)-v_{k+1}(t) \geq 0$; therefore, the assertion holds.
Lemma 3. Let the operator $F: C[0, T] \rightarrow C[0, T]$ be incremental and strictly increasing with constants $k_{1}, k_{2}$ as defined by Definition 1. Then,

$$
\begin{gathered}
\left\|\left(k_{2} v_{1}(t)-F\left[v_{1}\right](t)\right)-\left(k_{2} v_{2}(t)-F\left[v_{2}\right](t)\right)\right\|_{\infty} \\
\leq\left(k_{2}-k_{1}\right)\left\|v_{1}(t)-v_{2}(t)\right\|_{\infty}
\end{gathered}
$$

for all $v_{1}(t), v_{2}(t) \in C[0, T]$.

Proof. See [19].

Theorem 4. Consider a system of the form $y(t)=F[v](t)$. Let the operator $F: C[0, T] \rightarrow C[0, T]$ be incremental and strictly increasing. If the constant $0<\gamma \leq 1 / k_{2}$ and $v_{0}, v_{d} \in C[0, T]$, $v_{0}(t) \leq v_{d}(t)$ for all $t \in[0, T]$, then the iterative control algorithm converges; that is,

$$
\left\|v_{d}(t)-v_{k}(t)\right\|_{\infty} \leq \rho^{k}\left\|v_{d}(t)-v_{0}(t)\right\|_{\infty},
$$

where $\rho<1$ and $v_{k}(t) \rightarrow v_{d}(t)$ uniformly in $t \in[0, T]$ as $k \rightarrow \infty$.

Proof. To prove the convergence of the ILC algorithm, we show contraction of the input (2). Subtracting $v_{d}(t)$ from both sides of (2) and substituting $F[v](t)$ in place of $y(t)$, we get

$$
\begin{aligned}
v_{d}(t) & -v_{k+1}(t) \\
& =v_{d}(t)-v_{k}(t)-\gamma\left(y_{d}(t)-y_{k}(t)\right) \\
& =v_{d}(t)-v_{k}(t)-\gamma\left(F\left[v_{d}\right](t)-F\left[v_{k}\right](t)\right),
\end{aligned}
$$

for all $t \in[0, T]$. Taking the function $\|\cdot\|_{\infty}$ norm of (9), we get

$$
\begin{aligned}
\| v_{d}(t) & -v_{k+1}(t) \|_{\infty} \\
& =\left\|v_{d}(t)-v_{k}(t)-\gamma\left(F\left[v_{d}\right](t)-F\left[v_{k}\right](t)\right)\right\|_{\infty} .
\end{aligned}
$$

By Lemma 2, we obtain $v_{d}(t) \geq v_{k}(t)$ for all $t \in[0, T]$. Since the operator $F$ is incrementally strictly increasing with constants $k_{1} \leq k_{2}$ and $0<\gamma \leq 1 / k_{2}$, then by Lemma 3 we obtain

$$
\begin{aligned}
\| v_{d}(t) & -v_{k+1}(t) \|_{\infty} \\
& \leq\left(1-\gamma k_{1}\right)\left\|v_{d}(t)-v_{k}(t)\right\|_{\infty} \\
& \triangleq \rho\left\|v_{d}(t)-v_{k}(t)\right\|_{\infty}
\end{aligned}
$$

where $\rho=1-\gamma k_{1}<1$. Because $0<\rho<1$, (11) is a contraction. By induction, we obtain that

$$
\left\|v_{d}(t)-v_{k}(t)\right\|_{\infty} \leq \rho^{k}\left\|v_{d}(t)-v_{0}(t)\right\|_{\infty} ;
$$

therefore, the sequence $\left\|v_{d}(t)-v_{d}(t)\right\|_{\infty} \rightarrow 0$ as $k \rightarrow \infty$; that is, $v_{k}(t)$ converges to $v_{d}(t)$ uniformly in $t \in[0, T]$. Proof is completed. 


\section{The Prandtl-Ishlinskii Hysteresis Model}

The Prandtl-Ishlinskii (PI) model can be used to capture the rate-independent hysteresis nonlinearity in piezoelectric actuators. In this section, the PI model is presented.

The PI model utilizes the play or stop operators and a density function to characterize the hysteresis behavior. The hysteresis play operator is illustrated in Figure 2, while its detailed formulations have been presented in [20]. For a given input $v(t) \in C_{m}[0, T]$, the play operator $F_{r}[v](t)$ with threshold $r$ is defined by

$$
\begin{aligned}
F_{r}[v ; \psi](0) & =f_{r}(v(0), \psi), \\
F_{r}[v ; \psi](t) & =f_{r}\left(v(t), F_{r}[v ; \psi]\left(t_{i}\right)\right), \\
\text { for } t_{i} & <t \leq t_{i+1}, \quad 0 \leq i \leq N,
\end{aligned}
$$

with

$$
f_{r}(v, w)=\max (v-r, \min (v+r, w)),
$$

where $\psi$ is initial value of the operator $F_{r}$, and $0=t_{0}<t_{1}<$ $\cdots<t_{N}=T$ is a partition of $[0, T]$, such that the input function $v$ is monotone on each subinterval $\left[t_{i}, t_{i+1}\right]$.

\subsection{Property of Play Operator}

Lemma 5. For any $r \geq 0$, the operator $F_{r}$ can be extended uniquely to a Lipschitz continuous operator $F_{r}: C[0, T] \times \mathbb{R} \rightarrow$ $C[0, T]$. And it holds for all $v_{1}, v_{2} \in C[0, T]$, for all initial values $\psi_{1}, \psi_{2} \in \mathbb{R}$, and for all $t \in[0, T]$. Consider

$$
\begin{aligned}
& \left|F_{r}\left[v_{1} ; \psi_{1}\right](t)-F_{r}\left[v_{2} ; \psi_{2}\right](t)\right| \\
& \quad \leq \max \left(\sup _{\tau \in[0, t]}\left|v_{1}(\tau)-v_{2}(\tau)\right|,\left|\psi_{1}-\psi_{2}\right|\right) \\
& F_{r}\left[v_{1} ; \psi_{1}\right](t) \leq F_{r}\left[v_{2} ; \psi_{2}\right](t), \quad \text { if } v_{1} \leq v_{2}, \quad \psi_{1} \leq \psi_{2} .
\end{aligned}
$$

Proof. See [20] Section 2.3.

Lemma 6. If $v_{1}(t) \geq v_{2}(t)$, for all $t \in[0, T]$, and initial value $\psi_{1}=\psi_{2}=0$, a Lipschitz continuous operator $F_{r}: C[0, T] \times$ $R \rightarrow C[0, T]$ is incremental and strictly increasing; that is, there exist constants $k_{1}, k_{2}>0$ such that $k_{1}\left(v_{1}(t)-v_{2}(t)\right) \leq$ $F_{r}\left[v_{1}\right](t)-F_{r}\left[v_{2}\right](t) \leq k_{2}\left(v_{1}(t)-v_{2}(t)\right)$.

Proof. Consider

$$
\begin{aligned}
F_{r} & {\left[v_{1} ; \psi_{1}\right](t)-F_{r}\left[v_{2} ; \psi_{2}\right](t) } \\
& =F_{r}\left[v_{1}\right](t)-F_{r}\left[v_{2}\right](t) \\
& =f_{r}\left(v_{1}(t), F_{r}\left[v_{1}\right](t-1)\right)-f_{r}\left(v_{2}(t), F_{r}\left[v_{2}\right](t-1)\right) .
\end{aligned}
$$

Since $v_{1}(t) \geq v_{2}(t)$, for all $t \in[0, T]$, and initial value $\psi_{1}=$ $\psi_{2}=0$, then $F_{r}\left[v_{1}\right](0) \geq F_{r}\left[v_{2}\right](0)$. By induction, $F_{r}\left[v_{1}\right](t) \geq$ $F_{r}\left[v_{2}\right](t)$. There must exist a constant $k_{1}>0$ such that

$$
F_{r}\left[v_{1}\right](t)-F_{r}\left[v_{2}\right](t) \geq k_{1}\left(v_{1}(t)-v_{2}(t)\right) .
$$

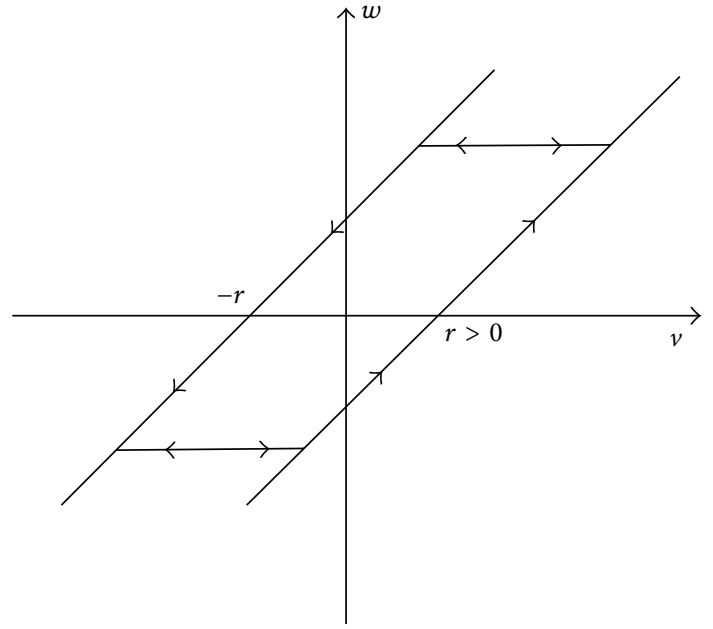

FIGURE 2: Play operator.

Because $F_{r}\left[v_{1}\right](t)-F_{r}\left[v_{2}\right](t) \geq 0$ and $\psi_{1}=\psi_{2}=0$, (15) can be simplified as

$$
F_{r}\left[v_{1}\right](t)-F_{r}\left[v_{2}\right](t) \leq \sup _{\tau \in[0, t]}\left(v_{1}(\tau)-v_{2}(\tau)\right)
$$

Let $\alpha(t)>0$ and $\sup _{\tau \in[0, t]}\left(v_{1}(\tau)-v_{2}(\tau)\right)=\alpha(t)\left(v_{1}(t)-v_{2}(t)\right)$. We get

$$
F_{r}\left[v_{1}\right](t)-F_{r}\left[v_{2}\right](t) \leq \sup _{\tau \in[0, t]}(\alpha(\tau))\left(v_{1}(t)-v_{2}(t)\right) .
$$

Let $k_{2}=\sup _{\tau \in[0, t]}(\alpha(\tau))$. From (18) and (20), we obtain

$$
\begin{aligned}
k_{1}\left(v_{1}(t)-v_{2}(t)\right) & \leq F_{r}\left[v_{1}\right](t)-F_{r}\left[v_{2}\right](t) \\
& \leq k_{2}\left(v_{1}(t)-v_{2}(t)\right) ;
\end{aligned}
$$

therefore, the assertion holds.

3.2. Property of the Prandtl-Ishlinskii Model. The PI model assumes that the output $y(t)$ of a piezoelectric actuator is a weighted superposition of basic $F_{r}[v]$. The output $y(t)$ is written as

$$
y(t)=H[v](t)=\int_{0}^{\infty} F_{r}[v](t) p(r) d r,
$$

where $p(r)$ is a given density function, satisfying $p(r) \geq 0$ with $\int_{0}^{\infty} p(r) d r<\infty$.

Lemma 7. If $v_{1}(t) \geq v_{2}(t)$, for all $t \in[0, T]$, and all initial values $\psi_{1}=\psi_{2}$ in all operators $F_{r}$, then PI operator $H$ : $C[0, T] \rightarrow C[0, T]$ is incremental and strictly increasing; that is, there exist constants $\lambda_{1}, \lambda_{2}>0$ such that

$$
\begin{aligned}
\lambda_{1}\left(v_{1}(t)-v_{2}(t)\right) & \leq H\left[v_{1}\right](t)-H\left[v_{2}\right](t) \\
& \leq \lambda_{2}\left(v_{1}(t)-v_{2}(t)\right)
\end{aligned}
$$

for any $t \in C[0, T]$. 


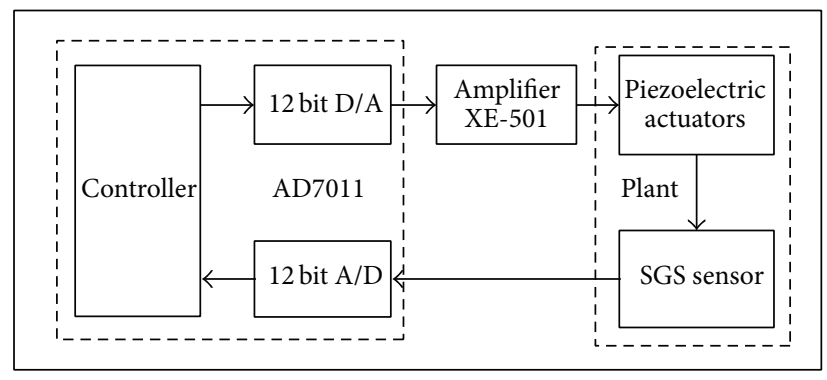

FIGURE 3: Structure of the experimental setup.

Proof. Consider

$$
\begin{aligned}
H\left[v_{1}\right] & (t)-H\left[v_{2}\right](t) \\
& =\int_{0}^{\infty} F_{r}\left[v_{1}\right](t) p(r) d r-\int_{0}^{\infty} F_{r}\left[v_{2}\right](t) p(r) d r \\
& =\int_{0}^{\infty}\left(F_{r}\left[v_{1}\right](t)-F_{r}\left[v_{2}\right](t)\right) p(r) d r \\
& \leq \int_{0}^{\infty} k_{2}\left(v_{1}(t)-v_{2}(t)\right) p(r) d r \\
& =k_{2} \int_{0}^{\infty} p(r) d r\left(v_{1}(t)-v_{2}(t)\right) \\
\triangleq & \lambda_{2}\left(v_{1}(t)-v_{2}(t)\right)
\end{aligned}
$$

for any $t \in C[0, T]$, where $\lambda_{2}=k_{2} \int_{0}^{\infty} p(r) d r$. Similarly, we can get

$$
\begin{aligned}
& H\left[v_{1}\right](t)-H\left[v_{2}\right](t) \\
& \quad=\int_{0}^{\infty}\left(F_{r}\left[v_{1}\right](t)-F_{r}\left[v_{2}\right](t)\right) p(r) d r \\
& \quad \geq k_{1} \int_{0}^{\infty} p(r) d r\left(v_{1}(t)-v_{2}(t)\right) \\
& \quad \triangleq \lambda_{1}\left(v_{1}(t)-v_{2}(t)\right)
\end{aligned}
$$

for any $t \in C[0, T]$, where $\lambda_{1}=k_{1} \int_{0}^{\infty} p(r) d r$. Proof is completed.

Theorem 8. Consider a hysteretic system of the form $y(t)=$ $H[v](t)$. Let the PI hysteresis operator $H$ satisfy the condition of Lemmas 6 and 7. If the constant $0<\gamma \leq 1 / \lambda_{2}$ and $v_{0}, v_{d} \in$ $C[0, T]$ in $(2), v_{0}(t) \leq v_{d}(t)$ for all $t \in[0, T]$, then the iterative control algorithm converges; that is,

$$
\left\|v_{d}(t)-v_{k}(t)\right\|_{\infty} \leq \rho^{k}\left\|v_{d}(t)-v_{0}(t)\right\|_{\infty},
$$

where $0<\rho<1$ and $v_{k}(t) \rightarrow v_{d}(t)$ uniformly in $t \in[0, T]$ as $k \rightarrow \infty$.

Proof. The proof is identical to the proof of Theorem 4 .

\section{Experimental Results}

4.1. Experimental Setup. The ILC algorithm is applied on an experimental piezoelectric actuator PST150/7/40VS12, which is a preloaded PZT from Piezomechanik in Germany. The natural frequency of the actuator is $20 \mathrm{kHz}$. The actuator provides a maximum displacement of $40 \mu \mathrm{m}$ and includes an integrated high-resolution strain gauge position sensor (SGS). The excitation module comprises a voltage amplifier (HVPZT XE-501.B) with a fixed gain of 15, which provides the excitation voltage for the actuator. The AD7011-EVA controller board equipped with 12-bit ADC and12-bit DAC is utilized to generate and acquire input voltage and output displacement signals. The experimental data are acquired at a sampling frequency of $1 \mathrm{kHz}$. Figure 3 illustrates the structure of the experimental system.

4.2. Experimental Result. We apply the ILC algorithm to track a sinusoidal trajectory $y_{d}(t)=5 \sin ((1 / 2 \pi) t-(\pi / 2))+7$. The constant $\gamma$ in (2) is chosen to be $\gamma=0.5$ and the initial input $v_{0}(t)=5 \sin ((1 / 2 \pi) t-(\pi / 2))+5$. The experimental results are shown in Figure 4.

Figure 4(a) shows the results of the ILC algorithm to track the desired trajectory, and the feedforward input of the ILC algorithm is depicted in Figure 4(b). The maximum error at the $k$ th iteration $\left\|e_{k}(t)\right\|_{\infty}$ is shown in Figure $4(\mathrm{c})$, and it demonstrated that convergence of ILC algorithm is achieved. Figure $4(\mathrm{~d})$ shows the tracking error at the 30 th iteration. The maximum error is approximately $0.015 \mu \mathrm{m}$, which is $0.15 \%$ of the total displacement range.

\section{Conclusions}

In this paper, we designed an ILC algorithm to compensate for hysteresis-caused tracking error in piezoelectric actuators and proved convergence of this algorithm on the whole tracking trajectory. Experiments were carried out to verify the effectiveness of the ILC algorithm. The experimental results show that the tracking error can be reduced to the noise level of the sensor measurements.

\section{Conflict of Interests}

The authors declare that there is no conflict of interests regarding the publication of this paper. 


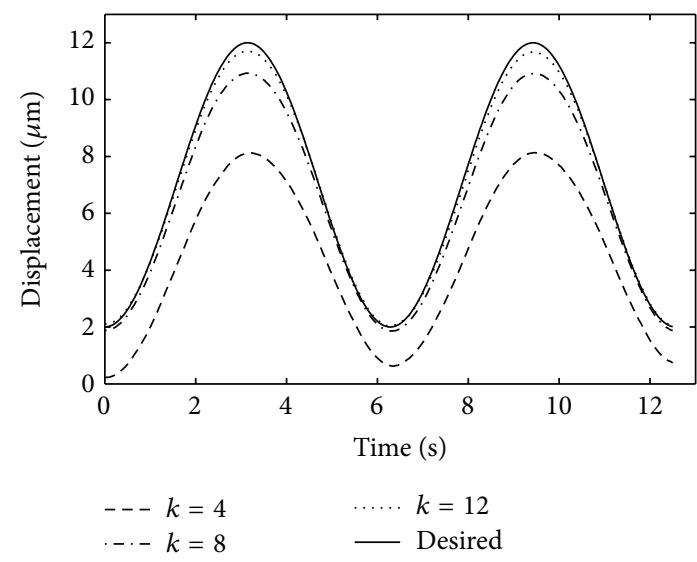

(a) Displacement versus time

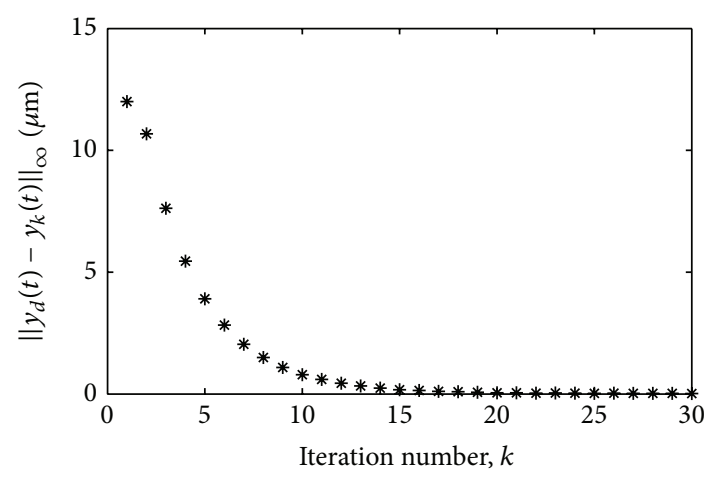

(c) $\left\|e_{k}(t)\right\|_{\infty}$ versus $k$

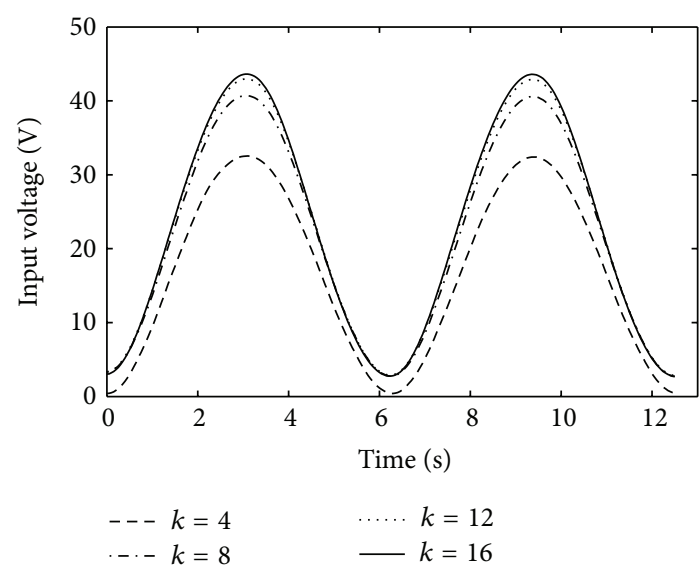

(b) Input versus time

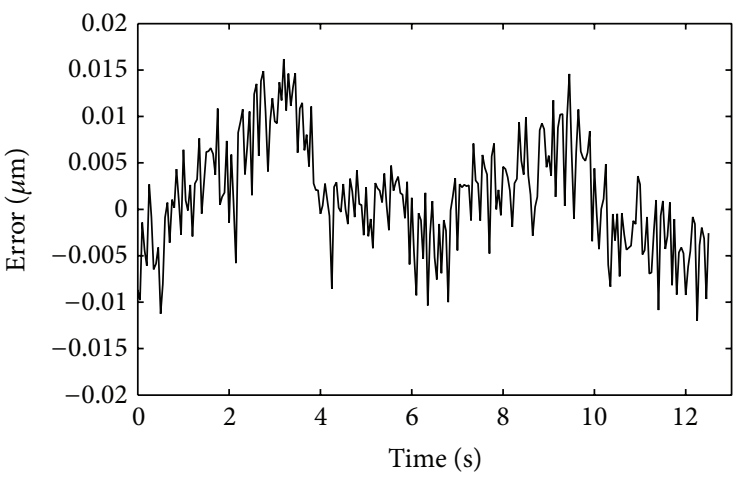

(d) $e_{30}(t)$ versus time

FIgURE 4: Experiment results.

\section{Acknowledgment}

This work is supported by the National Natural Science Foundation of China (no. 61174044).

\section{References}

[1] S. Devasia, E. Eleftheriou, and S. O. R. Moheimani, "A survey of control issues in nanopositioning," IEEE Transactions on Control Systems Technology, vol. 15, no. 5, pp. 802-823, 2007.

[2] M. Al Janaideh, S. Rakheja, and C.-Y. Su, "An analytical generalized prandtl-ishlinskii model inversion for hysteresis compensation in micropositioning control," IEEE/ASME Transactions on Mechatronics, vol. 16, no. 4, pp. 734-744, 2011.

[3] Q. Xu and P.-K. Wong, "Hysteresis modeling and compensation of a piezostage using least squares support vector machines," Mechatronics, vol. 21, no. 7, pp. 1239-1251, 2011.

[4] M. Al Janaideh and P. Krejci, "Inverse rate-dependent prandtlishlinskii model for feed forward compensation of hysteresis in a piezomicropositioning actuator," IEEE/ASME Transactions on Mechatronics, vol. 18, pp. 1498-1507, 2013.

[5] Y. Qin, Y. Tian, D. Zhang, B. Shirinzadeh, and S. Fatikow, "A novel direct inverse modeling approach for hysteresis compensation of piezoelectric actuator in feedforward applications," IEEE/ASME Transactions on Mechatronics, vol. 18, no. 3, pp. 981989, 2013.
[6] X. Tan and J. S. Baras, "Modeling and control of hysteresis in magnetostrictive actuators," Automatica, vol. 40, no. 9, pp. 1469-1480, 2004.

[7] H. C. Liaw, B. Shirinzadeh, and J. Smith, "Enhanced sliding mode motion tracking control of piezoelectric actuators," Sensors and Actuators A: Physical, vol. 138, no. 1, pp. 194-202, 2007.

[8] J.-X. Xu and K. Abidi, "Discrete-time output integral slidingmode control for a piezomotor-driven linear motion stage," IEEE Transactions on Industrial Electronics, vol. 55, no. 11, pp. 3917-3926, 2008.

[9] H.-J. Shieh and C.-H. Hsu, "An adaptive approximator-based backstepping control approach for piezoactuator-driven stages," IEEE Transactions on Industrial Electronics, vol. 55, no. 4, pp. 1729-1738, 2008.

[10] S. Bashash and N. Jalili, "Robust multiple frequency trajectory tracking control of piezoelectrically driven micro/nanopositioning systems," IEEE Transactions on Control Systems Technology, vol. 15, no. 5, pp. 867-878, 2007.

[11] P. Krejci and K. Kuhnen, "Inverse control of systems with hysteresis and creep," IEE Proceedings: Control Theory and Applications, vol. 148, no. 3, pp. 185-192, 2001.

[12] L. Sun, C. Ru, W. Rong, L. Chen, and M. Kong, "Tracking control of piezoelectric actuator based on a new mathematical model," Journal of Micromechanics and Microengineering, vol. 14, no. 11, pp. 1439-1444, 2004. 
[13] P. Ge and M. Jouaneh, "Tracking control of a piezoceramic actuator," IEEE Transactions on Control Systems Technology, vol. 4, pp. 209-216, 1996.

[14] X. Tan and J. S. Baras, "Adaptive identification and control of hysteresis in smart materials," IEEE Transactions on Automatic Control, vol. 50, no. 6, pp. 827-839, 2005.

[15] K. K. Leang and S. Devasia, "Iterative feedforward compensation of hysteresis in piezo positioners," in Proceedings of the 42nd IEEE Conference on Decision and Control, vol. 3, pp. 2626-2631, December 2003.

[16] K. K. Leang and S. Devasia, "Design of hysteresis-compensating iterative learning control for piezo-positioners: application to atomic force microscopes," Mechatronics, vol. 16, no. 3-4, pp. 141-158, 2006.

[17] R. V. Iyer, X. Tan, and P. S. Krishnaprasad, "Approximate inversion of the preisach hysteresis operator with application to control of smart actuators," IEEE Transactions on Automatic Control, vol. 50, no. 6, pp. 798-810, 2005.

[18] Y. Wu and Q. Zou, "Iterative control approach to compensate for both the hysteresis and the dynamics effects of piezo actuators," IEEE Transactions on Control Systems Technology, vol. 15, no. 5, pp. 936-944, 2007.

[19] R. Venkataraman and P. S. Krishnaprasad, "Approximate inversion of hysteresis: theory and numerical results," in Proceedings of the 39th IEEE Confernce on Decision and Control, pp. 44484454, December 2000.

[20] M. Brokate and J. Sprekels, Hysteresis and Phase Transitions, Springer, New York, NY, USA, 1996. 


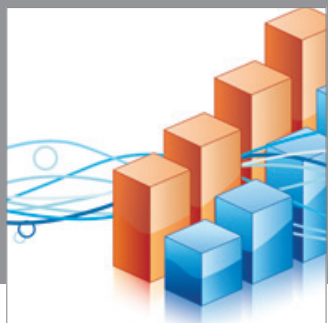

Advances in

Operations Research

mansans

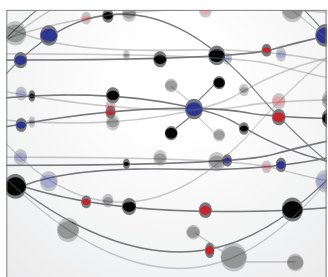

The Scientific World Journal
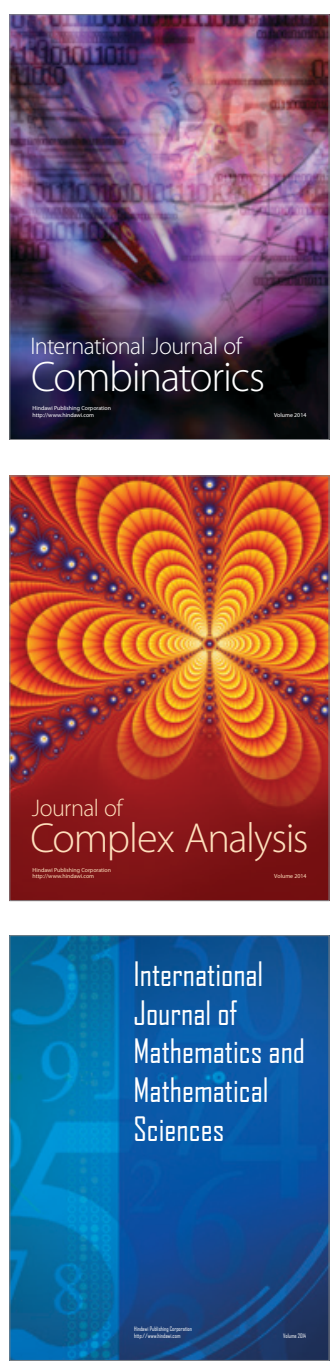
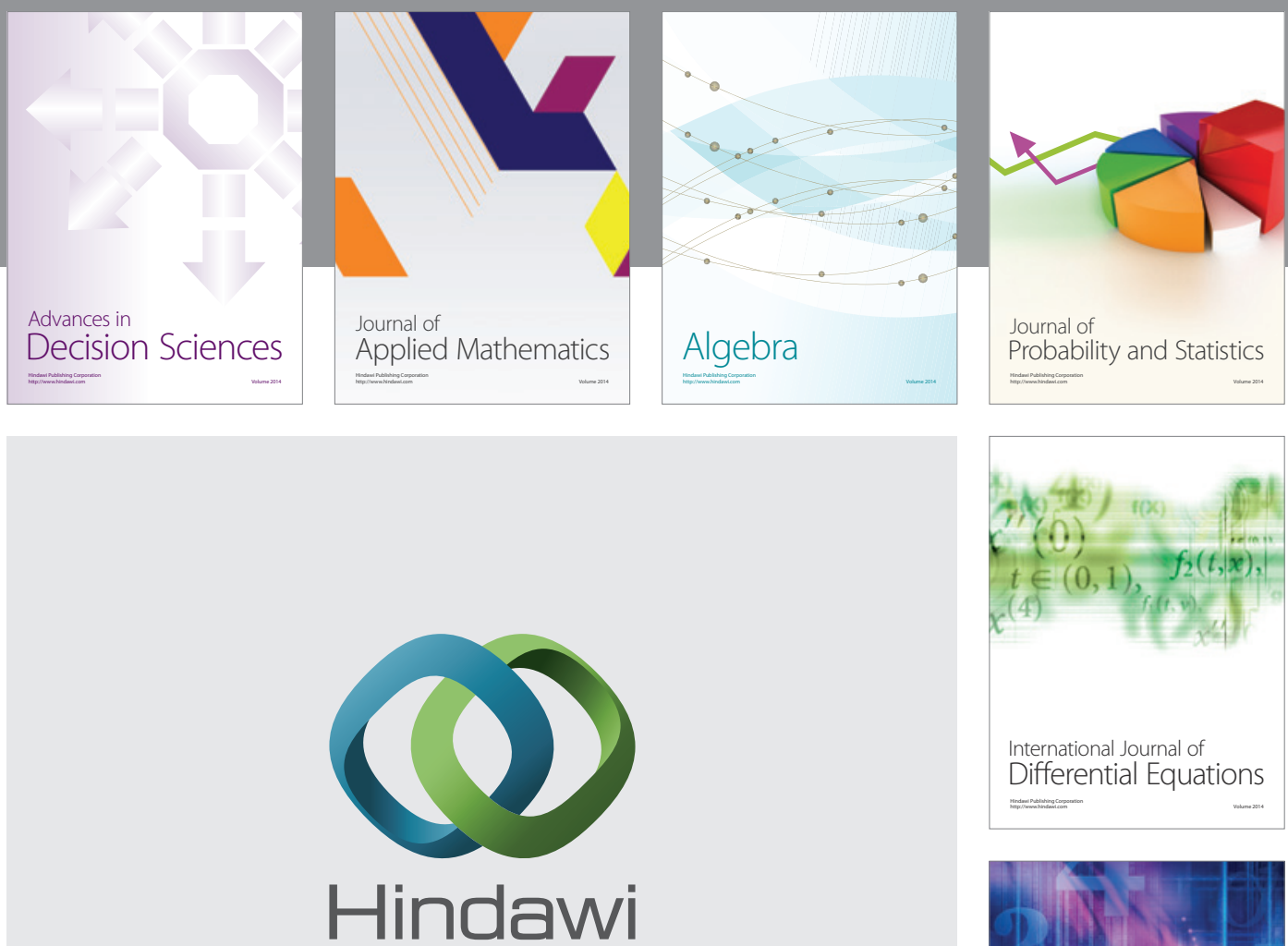

Submit your manuscripts at http://www.hindawi.com
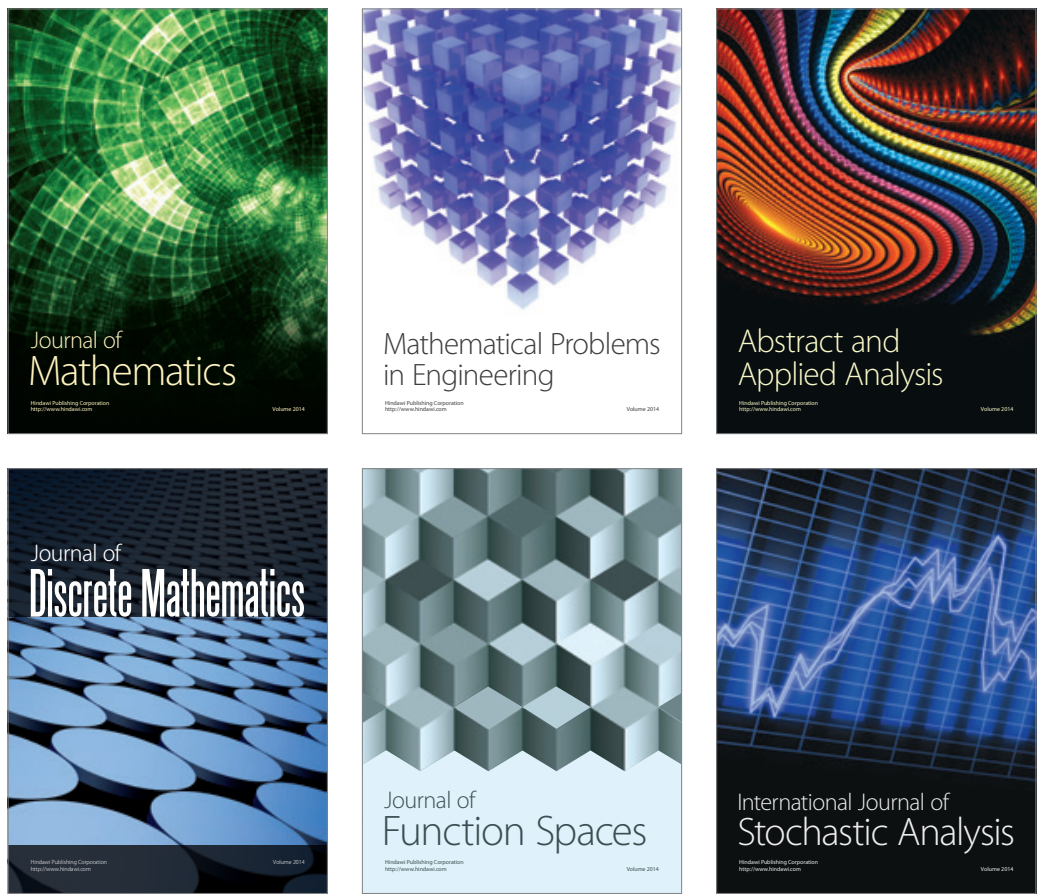

Journal of

Function Spaces

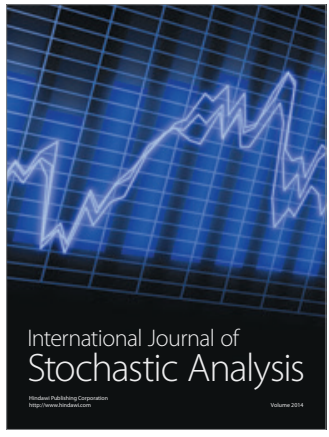

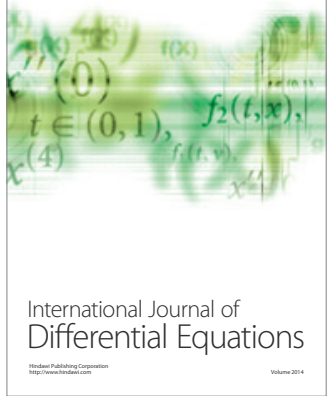
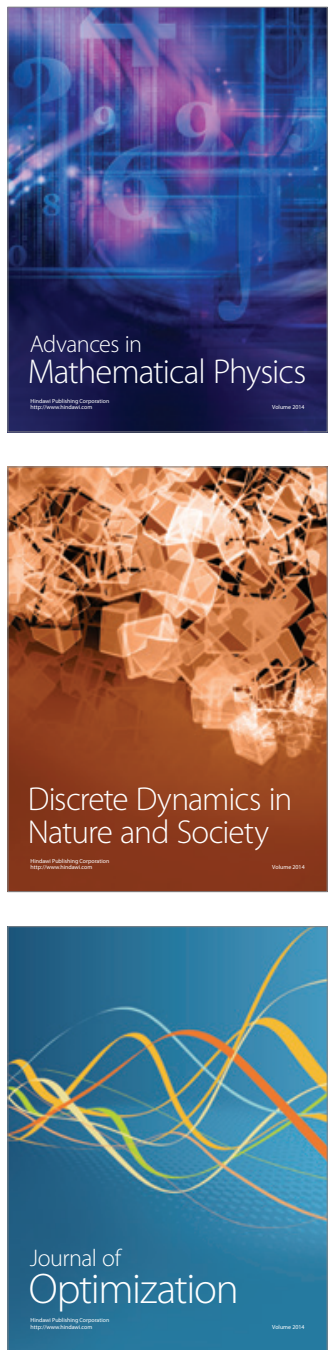